\title{
Removal of Artifacts from ECG Signal using RLS based Adaptive Filter
}

\author{
Runali S. Kamble \\ Dept. of ECE, BIT \\ Ballarpur, Maharashtra \\ 442701, INDIA
}

\author{
Sunil V. Kuntawar \\ Dept. of ECE, BIT \\ Ballarpur, Maharashtra \\ 442701, INDIA
}

\begin{abstract}
Artifacts cause the error in reading of ECG signals. The artifacts like PLI, Baseline wander, Electromyogram are introduced and hence removal of these artifacts is an important task in biomedical science. Adaptive filtering algorithms are evolving rapidly to eradicate noise. In this paper, the RLS technique in comparison with the LMS technology to remove the noise from the ECG signal is proposed. RLS algorithm is applied to the real ECG signal, collected from the MIT BIH database. The comparison will be done based on minimum mean square error, PSNR and coefficient correlating factor. Since, the RLS algorithm shows typically fast convergence as compared to LMS algorithm. From the result it is concluded that RLS based algorithm performance is superior to that of LMS based algorithm.
\end{abstract}

\section{Keywords}

Adaptive filter, ECG, RLS, LMS, PSNR, MMSE, PLI

\section{INTRODUCTION}

Electrocardiography is the process of recording the electrical activity of the heart over a period of time using electrodes which are placed on the skin the electrical changes on the skin that are generated from the heart muscle's electro physiologic pattern of depolarizing during each heartbeat. The magnitude of the heart's electrical potential is measured and is recorded over a period of time; the usual duration is of 10 seconds. The graph of voltage verses time produced is termed as electrocardiogram. [1]

Electromyogram, instrumental noise, motion artifacts. The causes of interference are explained by James et al. in [2]. The segregation of high resolution ECG signal from this contaminated signal is an important issue to investigate. There are various techniques which have been used for artifacts rejection from ECG. Conventional filters remove the artifacts up to some extent but these filters are static filters. These filters cannot update their coefficients with change in environment. Adaptive cancellers are used to handle non-stationary signals. The adaptive filter can adjust the filter coefficients according to the adaptive algorithm Syed Rehmam et al [3]

For the removal of noise from ECG a comparison between most commonly used filters techniques like Notch filters, FIR filters, IIR filters, Wiener filter, Adaptive filters algorithms LMS, NLMS, DLMS etc. is proposed in Rajesh Wagh et al [4]. The different adaptive filter algorithms LMS, RLS, NLMS are compared on the basis of implementation aspects, computational complexity and signal to noise ratio. It proves that the RLS algorithm is best to eradicate the noise in terms of improved SNR,
MMSE by Jyoti et al [5]. Kalman based least mean square filter is proposed by M. Sushmitha et al [6] for the removal of power line interference from the ECG signals. Kalman filter minimize the mean square error and removes the PLI. Here the operation principal of Kalman filter is described. The analysis of the performance of LMS and NLMS based adaptive filters design and simulation is presented where noise is removed by adaptive algorithm by establishing correlation between noise and its estimated value Divya et al [7].

Patch based method for rejection of artifacts from ECG signals but at the cost of computational complexity and slow convergence coefficient is described by Akansha Deo et al [8]. Discrete Wavelength Transform is used to eliminate 50HZs PLI from the signal and comparison is made with the Butterworth IIR notch filter. The proposed method has an effect of wavelet thresholding on the ECG reconstruction where an IIR notch when applied to ECG shows ringing effect. HAAR wavelet transform remove the noise but changes the shape of reconstructed waveform which prove that Daubechies $\mathrm{Db} 4$ wavelet transform method is best Prajakta S Gokhale et al [9]. A survey of different techniques used for the noise removal is studied where a comparison between Finite impulse response filter with different window and an Infinite impulse response filter is used for the removal of noise. The result indicates that Kaiser Window based FIR filters is having maximum efficiency Bhumika et al [10].

As biomedical signals are affected by noise a design of adaptive filter with a dynamic structure is explained. The dynamic filter in the first step decreases the error drastically and as the adaptation count increases error decreases as a function of logarithm. DSAF performs better as proposed by Ju-Won Lee et al [11].

A delayed LMS algorithm is proposed which is mostly implemented in hardware with performance degradation is not acceptable, a correction term is added but it increases the power consumption. To over this problem a retiming DLMS architecture is used and which result in responsive and less degradable system B. V, Hood et al [12].

For the denoising an NLMS algorithm is applied. The paper describes comparison between the method implemented previously and the proposed method. To cope with the complexity and convergence issues without any restriction tradeoff modified NLMS algorithm is proposed for the removal of noise from ECG Smita Dubey et al [13]. When input signal is stochastic then Least Mean Square algorithm gives good performance but for deterministic input signal Recursive Least Square algorithm gives better performance than LMS algorithm. In this paper we proposed an efficient RLS algorithm for 
the removal of noise. For the validation of the system the corrupted signal is passed through the LMS filter. Result analysis shows that RLS algorithm given better result compared to the LMS algorithm in terms of PSNR, MMSE and correlation coefficient.

\section{ADAPTIVE FILTER AND RLS ALGORITHM}

An adaptive filter modifies its frequency response automatically to improve the performance with some criteria as shown in Figure1.

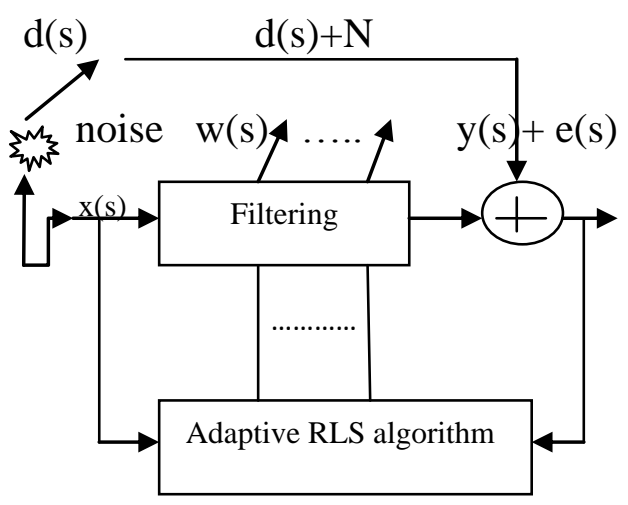

Fig.1. Adaptive filter structure with RLS algorithm

Due to its property to adjust to the changing environment adaptive filters are used in wide applications. Adaptive algorithm changes the coefficients of digital filter. LMS and RLS are basic algorithm. The LMS algorithm does not require the past information of the signal characteristics but provide filter coefficient estimation which progress with time. The RLS is based on steepest descent algorithm which changes its weight vector coefficient from sample to sample. It has fast convergence rate shows better performance. The coefficients of the RLS algorithm are as given.

$\mathrm{W}(\mathrm{n}+1)=\mathrm{w}(\mathrm{n})+\mathrm{e}(\mathrm{n}) \cdot \mathrm{k}(\mathrm{n})$

Where $\mathrm{w}(\mathrm{n})$ is the filter coefficient vector, $\mathrm{k}(\mathrm{n})$ is the gain factor, e(n) is error signal.

$\mathrm{k}(\mathrm{n})$ is given as

$\mathrm{k}(\mathrm{n})=\frac{p(n) \cdot u(n)}{\lambda+u^{t}(n) p(n) \cdot u(n)}$

$\lambda$ is the forgetting factor and $\mathrm{p}(\mathrm{n})$ is inverse correlation matrix and given as

$\mathrm{p}(\mathrm{n})=\delta^{-1} \mathrm{u}(\mathrm{n})$

Where $\delta$ is regulation factor and $\mathrm{u}(\mathrm{n})$ is unity matrix. The inverse correlation matrix in RLS algorithm is updated by [4] $e q^{n}$

$\mathrm{P}(\mathrm{n}+1)=\lambda^{-1}(\mathrm{Pn})-\lambda^{-1} \mathrm{k}(\mathrm{n}) u^{t}(n) \cdot \mathrm{P}(\mathrm{n})$
To adjust the RLS algorithm parameter one has to adjust forgetting factor. The forgetting factor lies in the range of 0 to 1 and the initial value of inverse correlation matrix is regulation factor.

\section{SIMULATION RESULT DISCUSSION}

The analysis of the system is performed on the MATLAB tool. The pure ECG signal which is applied as an input to the filter can be generated from MATLAB function of desired length or it can be collected from MIT-BIT Physionet database. The database contains 47 subjects ECG signal data. The data is collected from men and some of them are collected from women. Three records 109, 208,214 are randomly collected from 47 subjects. These signals are sampled at $360 \mathrm{~Hz}, 11$ bit over $10 \mathrm{mv}$ range of resolution. Then this noise free ECG is applied as an input and added with one of noise source such as PLI, baseline wander and eletroyogram. This is in turn applied to the adaptive filter as shown in Fig.2. The PLI is considered as noise which is added to the ECG while acquiring the ECG. This noise completely corrupts the original ECG signal, so it should be removed for the accurate diagnosis. For removal of noise it should be applied to above mentioned adaptive algorithms. Fig. 4(a) shows the original ECG signal. Then to this PLI is added as shown in Fig.4 (b) for 1450 samples. For the removal of noise an LMS algorithm is applied Fig.4(c) shows the denoised signal obtained after LMS filtering and a plot of minimum mean square error is obtained in Fig. 4(d). For the same record and for the same value of number of samples the RLS algorithm is applied to it and the graph is obtained in Fig.4 (e) along with MU plot in Fig. 4(f). From Fig.4(c) it is observed that some amount of noise is still present, but by using RLS algorithm this noise is removed almost. In the following figures $\mathrm{x}$ axis takes no of samples and $\mathrm{y}$ axis represent the amplitude. The same procedure is applied for the Baseline wander and electromyogram; outputs are obtained as plotted in Fig.5(a-f) and Fig.6(a-f) respectively. The PSNR obtained for LMS and RLS algorithm for PLI, Baseline Wander and electromyogram are estimated as shown in Table I. The performance characteristics in terms of MMSE and execution time are shown in Table II.

\section{CONCLUSION}

The proposed adaptive RLS technique gives an optimum quality of ECG signal. This paper mainly concentrates to reduce PLI, Baseline Wander and electromyogram using RLS based adaptive filters. The comparison of the proposed technique is made with the LMS algorithm. The performance analysis of the signal is done in terms of PSNR, MMSE and convergence rate. From the above analysis RLS algorithm gives better reduction of noise compared to LMS algorithm. The future development to this work can be made by implementation of wavelet based denoising for removal of base line wander and real time application of implemented algorithms. 

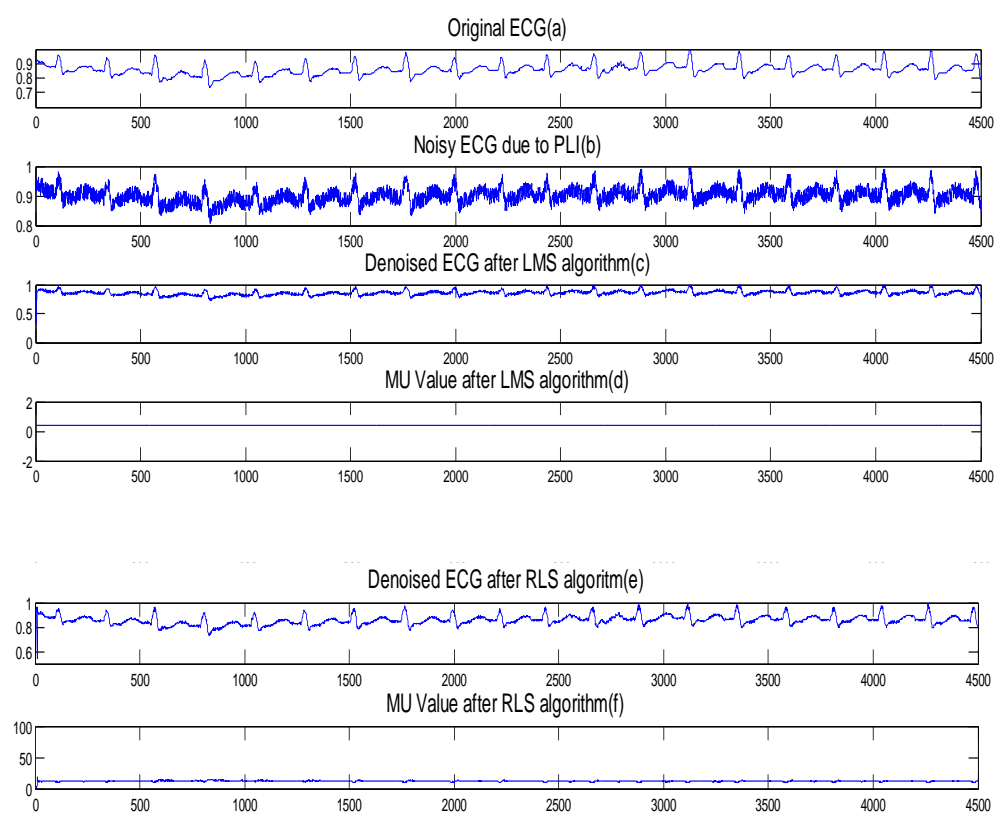

Fig 4: Plot of (a) MIT BIH original ECG record 109 with 4500 samples (b) ECG signal with PLI (c) Denoised signal using LMS algorithm (d) minimum min square error obtained by LMS algorithm (e) Denoised signal using RLS algorithm (f) minimum mean square error obtained by RLS algorithm
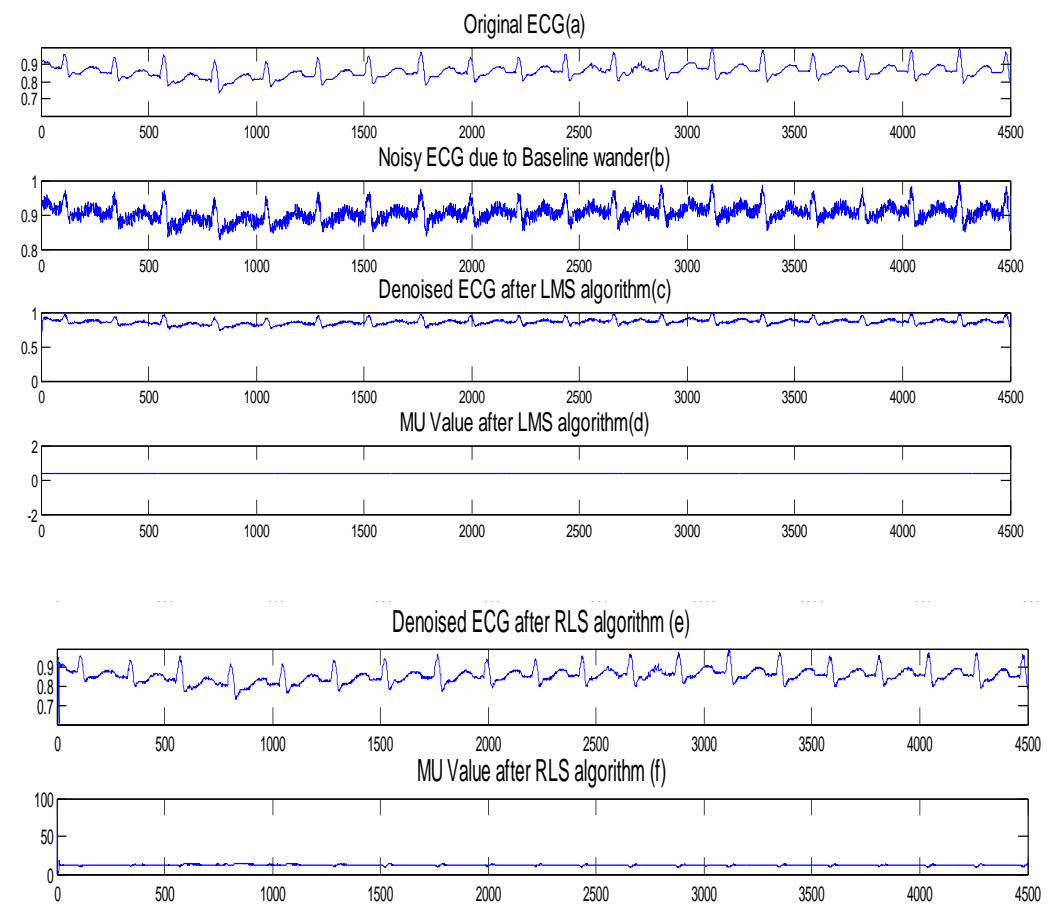

Fig 5: Plot of (a) MIT BIH original ECG record 109 with 4500 samples (b) ECG signal with Baseline Wander (c) Denoised signal using LMS algorithm (d) minimum min square error obtained by LMS algorithm (e) Denoised signal using RLS algorithm (f) minimum mean square error obtained by RLS algorithm 

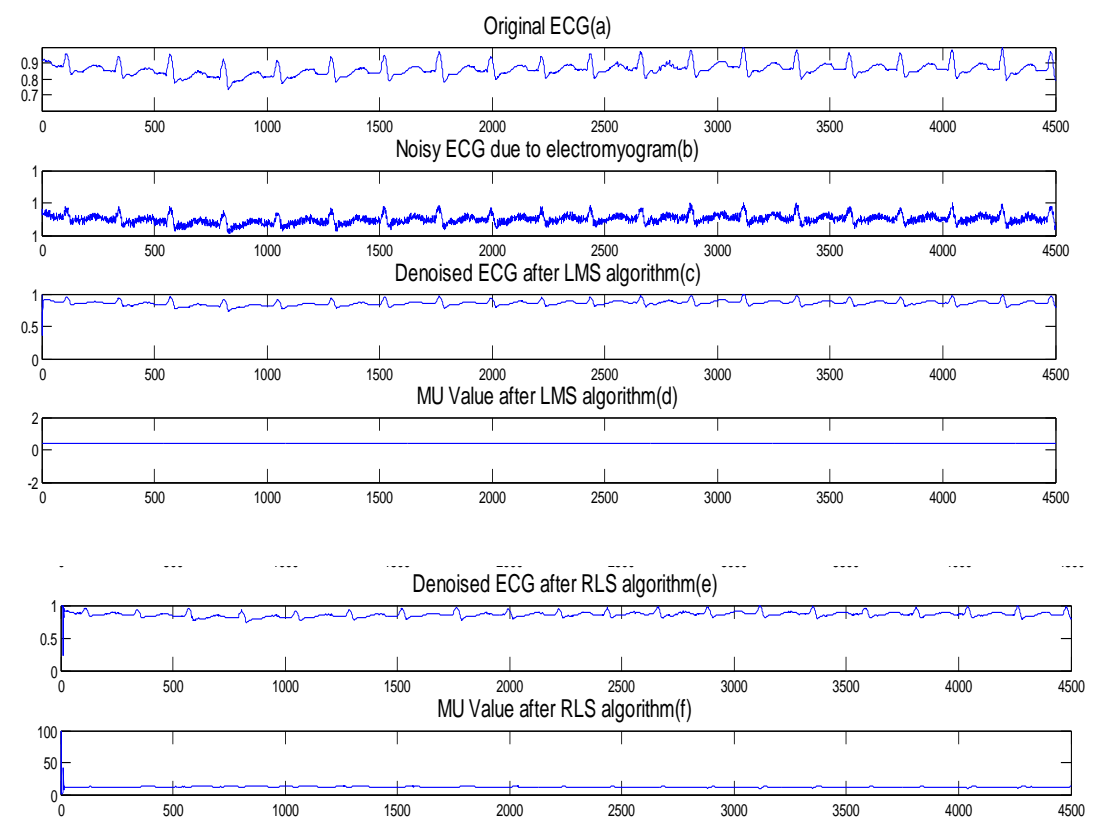

Fig 6: Plot of (a) MIT BIH original ECG record 109 with 4500 samples (b) ECG signal with Electromyogram (c) Denoised signal using LMS algorithm (d) minimum min square error obtained by LMS algorithm (e) Denoised signal using RLS algorithm (f) minimum mean square error obtained by RLS algorithm

Table 1: PSNR obtained after applying LMS and RLS algorithms

\begin{tabular}{|c|c|c|c|}
\hline $\begin{array}{l}\text { Algori } \\
\text { thm }\end{array}$ & Noise & $\begin{array}{l}\text { PSNR } \\
\text { before } \\
\text { filtering }\end{array}$ & $\begin{array}{l}\text { PSNR } \\
\text { after } \\
\text { filtering }\end{array}$ \\
\hline LMS & \multirow[t]{2}{*}{ PLI } & 30 & 35.5209 \\
\hline RLS & & 30 & 43.5292 \\
\hline LMS & \multirow[t]{2}{*}{ Baseline wander } & 30 & 36.9090 \\
\hline RLS & & 30 & 43.2813 \\
\hline LMS & \multirow{2}{*}{$\begin{array}{l}\text { Electromyogra } \\
\mathrm{m}\end{array}$} & 30 & 38.7472 \\
\hline RLS & & 30 & 38.9615 \\
\hline
\end{tabular}

Table 2: MMSE and EXECUTION TIME obtained after applying LMS and RLS algorithms

\begin{tabular}{|l|l|l|l|}
\hline $\begin{array}{l}\text { Algori } \\
\text { thm }\end{array}$ & Noise & MMSE & $\begin{array}{l}\text { TIME of } \\
\text { execution in } \\
\text { sec }\end{array}$ \\
\hline LMS & \multirow{2}{*}{ PLI } & 0.0002805 & 0.00156 \\
\cline { 1 - 2 } RLS & & 0.0004437 & 0.008681 \\
\hline LMS & \multirow{2}{*}{$\begin{array}{l}\text { Baselin } \\
\text { e }\end{array}$} & 0.00020375 & 0.001510 \\
\cline { 3 - 4 } & \multirow{2}{*}{ wander } & 0.00004697 & 0.007139 \\
\hline
\end{tabular}

\begin{tabular}{|l|l|l|l|}
\hline LMS & \multirow{2}{*}{$\begin{array}{l}\text { Electro } \\
\text { myogra }\end{array}$} & 0.000133 & 0.001497 \\
\cline { 3 - 4 } & $\mathrm{m}$ & 0.00012701 & 0.006909 \\
& & & \\
\hline
\end{tabular}

\section{REFERENCES}

[1] A. Muthuchudar, Lt.Dr.S.Santosh Baboo "A Study of the Processes Involved in ECG Signal Analysis" International journal of computer applications, March 2013, Volume 3, Issue 3,pp 1-5

[2] James C. Huhta, John G. Webster 1973 “ $60 \mathrm{~Hz}$ interference in electrocardiography" IEEE transactions on Biomedical engineering, vol 13, no.2, pp 91-101

[3] Syed Ateequr Rehaman and R Ranjith Kumar "Performance Comparision of Adaptive Filter Algorithm for ECG Signal Enhansment" IJARCCE, 2012, vol.1, issue 2, pp 86-90

[4] Rajesh D. Wagh, Kiran R. Khandarkar, Dipanjali D. Shipne, Shaila P. Kharde "Noise Removal from Electrocardiogram (ECG) a comparison Approaches" international journal of advance research in computer engineering \& technology,January 2014, vol 3, issues 1, pp 47-51

[5] Jyoti Dhiman, Shadab Ahmad, Kuldeep Gulia "Comparison between Adaptive filter Algorithms (LMS, NLMS and RLS)" International journal of science engineering and technology research, May 2013, vol 2, Issue 5, pp 1100-1103

[6] M. Sushmitha, T. Balaji "Removing the power line interference from ECG sigml using adaptive filters" international journal of computer science and network security, Nov 2014, vol 14, no 11, pp 76-79 
[7] Divya, Preeti Singh, Rajesh Mehra "Performance Analysis of LMS \& NLMS Algorithms for noise cancellation" sep 2013, vol 2, issue 6, pp 366-369

[8] Akanksha Deo, DBV Singh, Manoj Kumar Bandil, A K Wadhwani "Denoising of ECG signals with Adaptive filtering algorithm \& patch based method" international journal of computer network and wireless communication, June 2013, vol 3, No 3, pp 300-305

[9] Prajakta S Gokhale "ECG signal Denoising using Discrete Wavelet transform for removal of $50 \mathrm{~Hz}$ PLI Noise" international journal of emerging technology and advance engineering, May 2012, vol 2, Issue 5, pp 81-85

[10] Bhumika Chandrakar, O.P. Yadav, V.K. Chandra "A Survey of noise removal techniques for ECG signals" international journal of advance research in computer and communication engineering March 2013, vol 2, issue 3, pp 1354-1357

[11] Ju-Won Lee, Gun Ki Lee "Design of an adaptive filter with a Dynamic structure for ECG signal processing" international journal of control automation and system, March 2005, vol 3,no 1, pp $137-142$

[12] B.V. Hood, R.N.Mandavgane, J.D Dhande “ Retiming of delayed least mean square algorithm for adaptive filter: A Review" international journal for scientific research and development, 2016, vol 3, issue 11, pp 614-617

[13] Smita Dubey, Swati Verma "Denoising of the ECG signal using NLMS adaptive filtering algorithm" international journal of advance engineering research and studies, 2015, vol 4, issue 2, pp 343-345

[14] MIT-BIH Arrhythmia Database, www.physionet.org 